\title{
A system for automatically analyzing and correcting real-time experiment control programs
}

\author{
YORK MAKSIK and RUSSELL M. CHURCH \\ Brown University, Providence, Rhode Island
}

\begin{abstract}
A system is described that automatically analyzes the time constraints in a real-time experiment control program and automatically makes corrections to that program to provide any degree of temporal accuracy desired by the experimenter within the capabilities of the hardware. A generalized procedure is presented to allow similar systems to be developed for most common languages and hardware platforms.
\end{abstract}

Most of the programs that psychologists use to control experiments and record data cannot be tested formally to guarantee any particular level of performance. The problem is that the real-time programs that psychologists typically use are difficult or impossible to analyze formally to guarantee that all input events will be processed and all output events delivered within some specific time criterion. As a result, some events may not be handled in a timely manner, and others may be missed entirely.

The usual testing procedure for real-time programs that control experiments and record data is quite informal. It consists of running each program several times with different inputs, in the hope that some of these instances will be similar to the worst case that the program will encounter. The investigator then looks at the output and judges whether or not it appears to be sensible.

To analyze a typical experiment control program, it is necessary to have very detailed knowledge about the hardware and processor, the internal details of the language compiler or interpreter, and the worst-case time demands created by each subject's input and the corresponding contingencies. Of course, any change in the program would require a new analysis. When the difficulties of formally analyzing a real-time program are considered, informal testing procedures appear to be the only ones that are feasible.

\section{Our Requirements for a Real-Time \\ Experiment Control System}

In spite of the apparent difficulty of developing a programming system that would be analyzable, we were concerned about losing responses and incorrectly timing events as we switched from an old, well-tested environment hosted by a DEC PDP-11 to a new one hosted by an IBM PC/AT. We required a system that was suitable for many different types of experiments, that could con-

This research was supported by Grant MH-44234 from the National Institute of Mental Health and Grant BNS 9110158 from the National Science Foundation. Correspondence should be addressed to either author at the Department of Psychology, Box 1853, Brown University, Providence, RI 02912. trol many different experiments at the same time, and that could be easily programmed by many investigators. In addition to these standard requirements for a real-time control program, we also wanted to guarantee that (1) all time-critical events would be processed in the order of their occurrence within a short time limit-the critical limit specified by the investigator; (2) all other processing and analysis of data would be completed within a longer time limit-the noncritical limit specified by the investigator; and (3) any user code that might result in the violation of either of these time limits would be automatically detected and fixed if possible.

\section{The Real-Time Constraints Theorem}

The temporal constraints of a program can be analyzed and the three requirements above can be met if the control system meets the two conditions of the Tanenbaum realtime constraints theorem (Tanenbaum, 1987): (1) There must be a predictable, minimum constant amount of free processor time within each time segment that we are calling the critical limit, and (2) any procedure or program must be configurable as a state-transition system. If these two conditions are met, the system is formally analyzable under worst, best, and average conditions through the application of a standard cyclic search algorithm, a type of depth-first search.

\section{Satisfying The First Condition}

The first condition of the real-time constraints theorem states that a minimum predictable amount of free processor time must be available in any chosen time interval. If we choose a 1-msec interval as the critical limit, we must guarantee, in the worst case, that all events will be retrieved and processed within $1 \mathrm{msec}$ and that there will also be some minimum free processor time. This ensures that no backlog of processing can desynchronize the system (Tanenbaum, 1987). In Figure 1, notice the 1-msec interval labeled "critical limit," which consists of two parts: time for event processing and retrieval, and time for noncritical processing.

This first condition of the real-time constraints theorem has some strong implications regarding what kind of event 


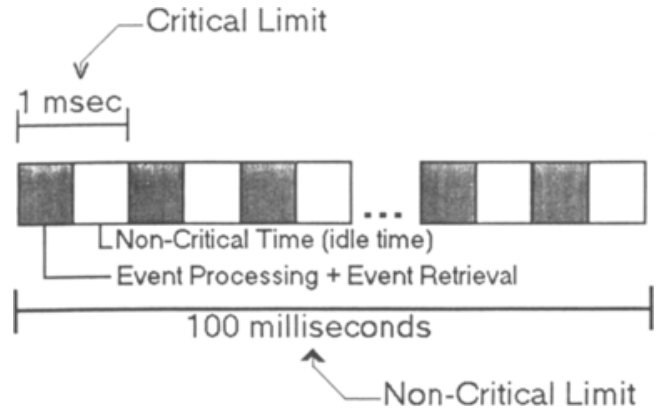

Figure 1. The critical and noncritical limits. The investigator has specified that all critical code (event retrieval and processing) be executed within $1 \mathrm{msec}$ (the critical limit) and that all other code in a single state be executed within $100 \mathrm{msec}$ (the noncritical limit). Thus all the code in the longest state must be executed in the amount of idle time available in $\mathbf{1 0 0} \mathbf{~ m s e c}$.

retrieval system is used; specifically, it forbids the two most commonly used event retrieval systems-simple polling and event-based interrupts.

A simple polling program sequentially examines the inputs to the system as quickly as possible, servicing any input or event as soon as it is detected. The other processing is also in this circular queue, and, if there is other work to be done, it will be completed before the program continues to poll the inputs. A process diagram for a simple polling system is shown in Figure 2. The Xs at the top represent responses waiting to be processed. If a particular response occurs several times in rapid succession, some responses may be lost or processing may be seriously delayed; the responses and events may also be processed out of order. There is no guaranteed minimum time to process a response, because that depends upon how many other inputs have recently occurred, how much processing needs to be done for each of them, and how much processing needs to be done for other purposes. A simple polling system will not separate other critical processing from noncritical processing, so there is no predictable minimum amount of idle time. Yet in spite of these problems in a multiprocessing environment, simple polling is the most efficient way of collecting data from a single input because it has minimum overhead.

An event-based interrupt program remains in the idle state, performing non-time-critical tasks until a response occurs. The program is interrupted, goes to an interrupt handler to service the input, and then immediately returns to the noncritical, idle state. Figure 3 shows a process diagram of such a system. If a particular response occurs several times in rapid succession, some responses may be lost. If several different responses or events occur in rapid succession, the responses and event may be processed out of order. (Of course, special buffering hardware could be added to alleviate this problem.) There is no guaranteed minimum time to process a response, since that depends on how many other inputs have recently occurred and how much processing needs to be done for each of them. Although normally it will be ample, a response-based interrupt program will not ensure a predictable amount of idle processor time in every chosen time segment. Thus, a formal analysis of time constraints is not possible. In cases in which a formal analysis of time constraints is not needed, the response-based interrupt system may be the best choice, because it gives optimal priority to the most time-critical events.

Thus, neither simple polling nor event-based interrupt systems provide a way to guarantee a minimum amount of idle time and thus are not suitable for formal analysis. We, therefore, chose to implement clock-based polling. It is an old and well-known method of collecting responses from input lines by computer. However, it is not used in many psychology labs, because it requires that the programmer gain precise control of the system clock or purchase an external clock that produces an interrupt. This is difficult on some modern personal computers such as most Apple Macintosh computers. It should be noted that software libraries are available to make system timer control extremely easy. ${ }^{1}$

A clock-based polling system is shown in Figure 4. With this system, the investigator chooses some short time limit within which all time-critical events are to be timedthe critical limit. We chose $1 \mathrm{msec}$ and verified that it would be possible to execute all of our instructions in that time by means of an automated method that we will explain in detail later. That interval is then subdivided into three sections: event retrieval, event processing, and available processor time for use in noncritical tasks. The system clock interrupts once every chosen time interval. When the processor receives this interrupt, it scans all the input lines once and places the number of each active line in a queue. As soon as all of the lines have been scanned and all relevant inputs have been placed in a queue, the computer enters the event-processing stage. Here the computer removes one number at a time from the queue and goes to a corresponding subroutine. Once all numbers have been removed from the queue and all subroutines have been executed, the computer can pro-

\section{Simple Polling}

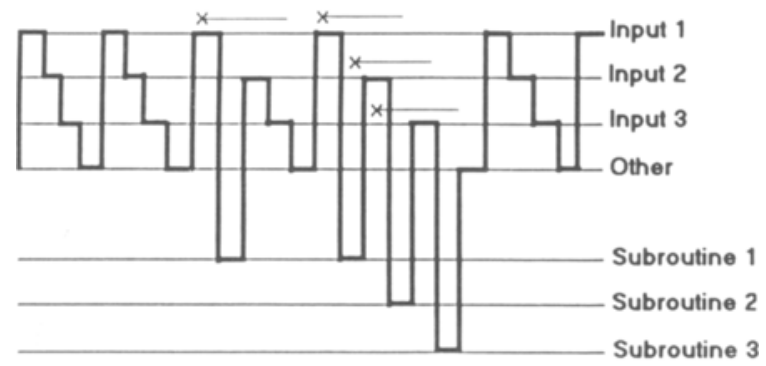

Time

Figure 2. A diagram of a simple polling system with three inputs and their associated subroutines. The program sequentially examines the inputs and other instructions as rapidly as possible. 


\section{Event Based Interrupts}

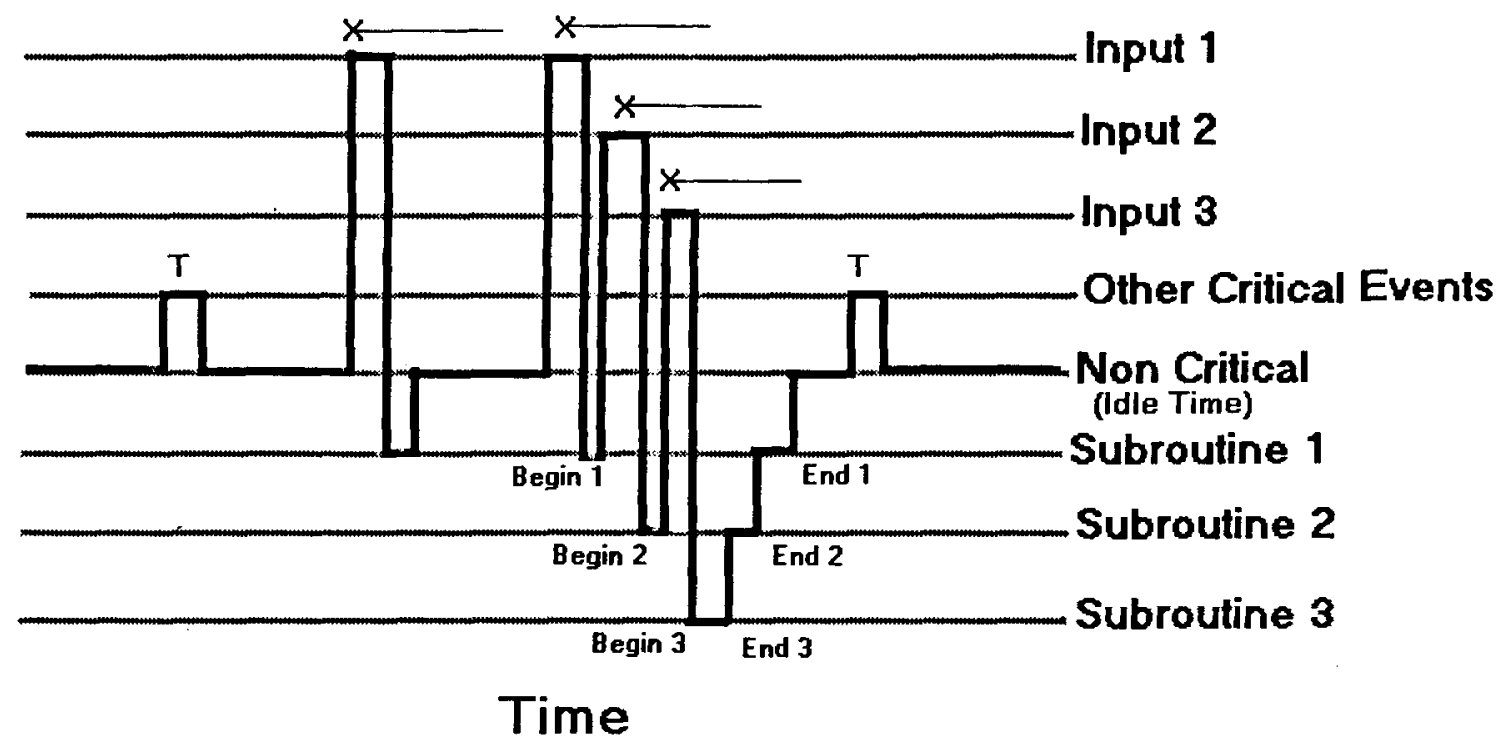

Figure 3. A diagram of an event-based interrupt system with three inputs and their associated subroutines. The program processes noncritical instructions until an input or another critical event (usually a clock, represented as a $T$ in the diagram) interrupts the program and is serviced.

cess less critical things, such as planning the next trial, or doing on-line data analysis while it waits for the next clock pulse telling it to retrieve time-critical events and responses again.

In our system, the worst case would be all 30 inputs active in each millisecond. Retrieving all 30 inputs in each interval requires only . $3 \mathrm{msec}$ on an IBM PC/AT ( 80286 running at $12 \mathrm{MHz}$ ), and executing all of our associated subroutines took only $.2 \mathrm{msec}$. This leaves half the processor time, $.5 \mathrm{msec}$ in each millisecond, free for less critical duties.

This method is reasonably efficient in its use of processing time, although it is not maximally efficient. Its main virtue is that it maintains the temporal stability of a system so that any analysis of a user program will hold true in practice. Also, it satisfies the first condition of the real-time constraints theorem.

\section{Satisfying the Second Condition}

The second condition of the real-time constraints theorem states that any program that is to be analyzed formally needs to be configured as a state-transition system. The reason for this is simple. A state-transition system contains strictly linear code within each state and has no internal loops that can halt the forward flow of a program. The analysis method that we use simply matches the syntactic patterns of a written program in a given language with a table of how much time a particular instruction takes. If one of those instructions is a loop, such as a FOR-NEXT loop in BASIC or a do-while loop in C, the analysis program cannot tell how long it will take to exit that loop if the loop depends on variables that are not defined until run time. Nearly all loops can be expressed as a state transition, in which, at the end of a given state, if a condition is not satisfied, the state simply goes to itself.

If we choose a 100-msec interval as the noncritical limit, we must guarantee, in the worst case, that all noncritical instructions will be executed within $100 \mathrm{msec}$. (See the 100 -msec interval labeled "noncritical limit" in Figure 1.) The sum of all idle time within that interval must be sufficient for all processing in the state that requires the most time. Because the minimum amount of noncritical time in each time segment has already been calculated and the maximum time required in each state can be calculated, the maximum amount of time to execute all noncritical instructions can be readily determined.

A segment of a small program written in C looked like this:

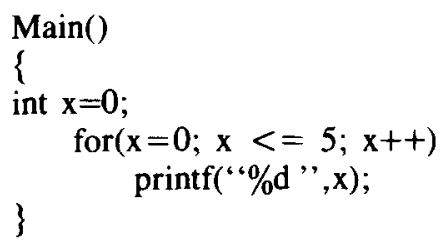

It could be rewritten as a state-transition system like this: 
int $x=0$;

STATE 1:

printf(" $\% \mathrm{~d}$ ", $\mathrm{x})$;

$\mathrm{x}++$;

if $(x<=5)$

goto STATE 1;

else

exit();

End STATE 1

Both programs accomplish the same task of counting from 0 to 5 and printing the results, but there are no loops to confuse the analyzing program in the middle of STATE 1. Notice also that if it was determined that STATE 1 was too long to execute in one large chunk, then it could easily be broken into two chunks as follows: int $x=0$;

STATE 1:

printf( “\%d", $\mathrm{x})$;

$\mathrm{x}++$;

goto STATE 2;

End STATE 1

STATE 2:

$$
\begin{aligned}
& \text { if }(x<=5) \\
& \text { goto STATE } 1 \text {; }
\end{aligned}
$$

End STATE 2;

The version with the for loop cannot be so easily broken up. Figure 5 shows a simple reaction time experiment written in a state-transition style. In general, any program that can be diagrammed like Figure 5 can be turned into a state-transition system. It consists of one or more states-in this case, two states labeled START

\section{Clock Based Polling}

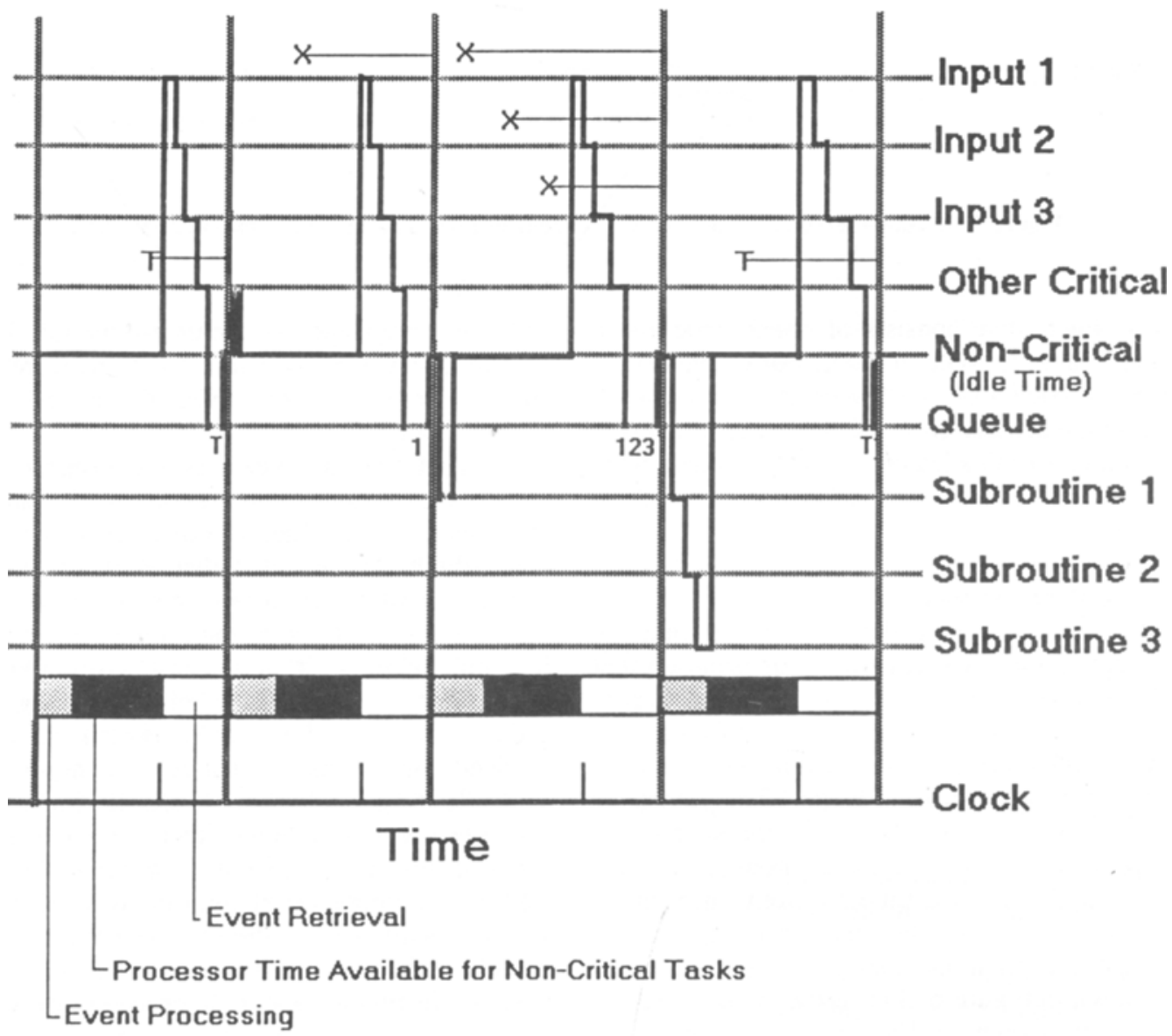

Figure 4. A diagram of a clock-based polling system with three inputs and their associated subroutines. The program processes noncritical instructions until a clock interrupts the program at some regular, short interval. The program sequentially examines the inputs, which are put on a queue and processed; then the program returns to noncritical instructions. 
User's Code

\author{
*sel NONCRITICAL 100 \\ \#set CAITICAL 1 \\ int responses_count $=0$;
}

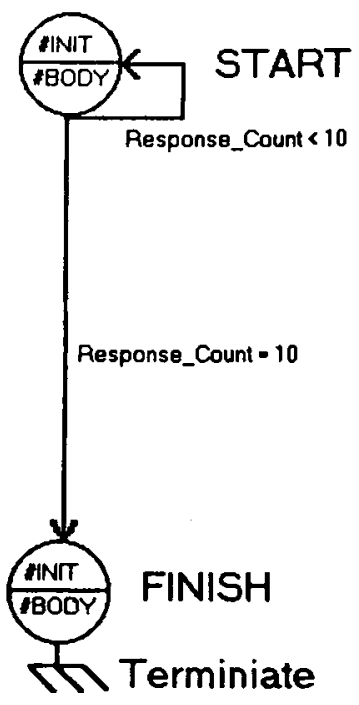

START:

\#init

\#body cls():
printf("Go"):
if (keypress())

break;

FINISH:

minih

ubody

printf("All Done");
Comments

* State 1 is named START. *

$r$ We further subdivided our states into $\%$

r. 2 parts, winit and "body. "\%

$r$ Clear the subjects screen $\because$

$r$ Print start message ."

$r$ Keypress() is a hardware routine we added ."

$r$ Increment trial counter . $\%$

r $\| 10$ responses then end session $\%$

$r^{\circ} \mathrm{go}$ to "zinit portion of state called FINISH ."

rothenwise go to thody portion of START. "

$r$ End state called START $\because$

$\ulcorner$ Begin state 2 called FINISH .

$r$ Tell subject he is finished ."

rEnd state called FINISH. \%

Figure 5. A sample program expressed as a state-transition diagram, with user-written code.

and FINISH. Each state consists of linear code and a branch to either another state or itself. For convenience, we have divided each state into two parts: initialization and body. If entry is made from another state, it is made into the beginning of initialization. If entry is made from the same state, a self-reference, it is made into the beginning of body.

\section{An Analyzing Preprocessor}

We developed our analysis and correction system in the form of a preprocessor, a small translator program that would read in code written in a superset of the $C$ programming language. This user-written code differed from $C$ in that we defined states instead of functions, and executed state transitions instead of loops. ${ }^{2}$ This translator program read in our user-written code for the experimental procedure and produced somewhat modified $\mathrm{C}$ code along with an analysis of the time required for each instruction, as well as of the total time for all critical and noncritical instructions in each state. Sample input and output are shown in Figure 6. This is the simple reaction time experiment shown in Figure 5, along with the preprocessor analysis and two other files that will be explained later. Notice that we had previously informed the preprocessor which instructions we thought were critical and thus were subject to the user-definable 1-msec critical limit, as well as which instructions were less critical and thus were subject to the user-definable 100 -msec noncritical limit.

The first step in developing our preprocessor was to decide what language features we needed and what currently available commercial compiler would best approximate them. We decided that Borland $\mathrm{C}$ was a good choice, and we added some special hardware control functions and internupt processing routines to the language before implementing it as a state-transition system.

The next step was to determine how long each line of $C$ code would take. Fortunately, Borland provided us with a diskette, upon request, that gave the number of processor cycles required for each $\mathrm{C}$ primitive and library function. Once we had obtained that, all we needed to know was what processor we were using and at what clock speed we were running it (an 80286 running at $12 \mathrm{MHz}$ ). Other companies are often willing to distribute timing information about their compiler outputs for a nominal fee. However, if information about the processor cycles needed to complete each line of a high-level language is not readily available, one can usually compile to assembly language and use a table on diskette distributed by Intel for their processors, or one distributed by Motorola for theirs. 


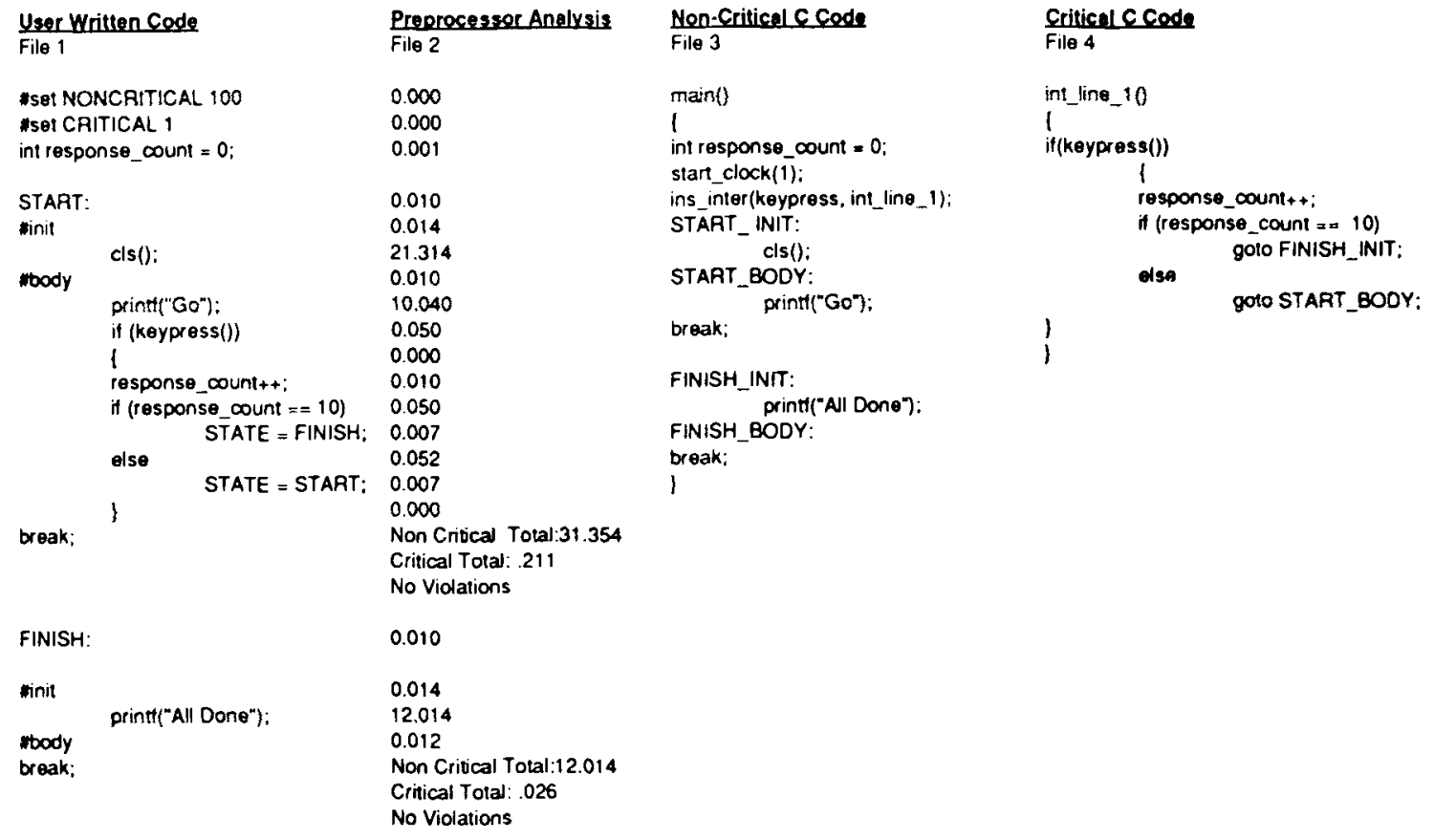

Figure 6. Analysis of the sample program with user-written code (File 1) and the time required for each instruction and all critical and noncritical instructions in each state (File 2). The preprocessor translates the user-written code to time-noncritical $C$ code (File 3 ) and time-critical $C$ code (File 4) and adds some initialization routines to both as needed.

We now had all of the ingredients needed to make a preprocessor: code translated to a form with no internal loops, and a way to measure the amount of time that it would take to execute each line of code.

The general algorithm is a simple search through the user's code (File 1, first column of Figure 6), line by line, in which each line of code is pattern matched with the keywords provided on the compiler manufacturer's diskette of language keywords. Each keyword has an associated number of processor cycles that are required to execute that line of code. The number is different for different processors and for different compilers. Once this number is retrieved, it is multiplied by the rate at which the user's computer executes processor cycles (typically microseconds/cycle or nanoseconds/cycle). This is the time to execute a line of user-written code in milliseconds.

After the amount of time needed to execute a particular line of code has been calculated, the line is compared against a previously defined list of critical or noncritical code. If the line is considered critical, the calculated time of execution is added to a variable containing the time used in this state for critical code. If the line is considered noncritical, the calculated time of execution is added to a variable containing the time used in this state for noncritical code.

After the time calculation is made and it has been decided whether or not that particular line of code is critical or noncritical, the preprocessor outputs the calculated execution time to a second file (File 2, second column of Figure 6), which will contain the time required for ex- ecution of each line of user's code. The preprocessor also takes that line of code and inserts it into either a file of noncritical code (File 3, third column of Figure 6) or a file of critical code (File 4, fourth column of Figure 6).

At the end, the preprocessor has translated the user's code into three files. One is the analysis of time constraints of the user's program, the second is the noncritical code, and the third is the critical code, which can be made into interrupt service routines for the clock tick interrupt.

Because the actual code is very long, below we provide an English pseudocode schematic of the algorithm that we used (and that we have described above). ${ }^{3}$ It is essentially a scaled-down language parser (Aho \& Ullman, 1977). 4

Begin

Read in a line of user written code (File 1 of Figure 6)

If the line is the name of the beginning of a state Begin

Output a function definition (or label marker) of the same name to the noncritical code file (File 3 in Figure 6),

Write out the function initialization code to the noncritical code file (File 3 in Figure 6).

Initialize the noncritical processing time counter. Initialize the critical processing time counter. End

If it is not the beginning of a state Begin 
If it is the end of a state

Begin

Output the value of the noncritical time counter

to the file where the analysis will be stored (File 2 in Figure 6).

Output the value of the critical time counter to the analysis file (File 2 in Figure 6).

Write out the code necessary for function termination in your language to the noncritical code file (File 3 in Figure 6).

End

If the line read is not the end of a state

Match the line to the keywords on the manufacturer's compiler information diskette.

Retrieve from the table on the diskette the number of processor cycles required to execute that line of code.

Multiply that number times the number of milliseconds per processor cycle that your processor can handle.

Add the result to either the critical or the noncritical time count, whichever is appropriate, by matching the keyword to commands that were defined as critical or noncritical.

End

If the line was marked as critical

Output it to the file of time-critical code (File 4 in Else Figure 6)

Output it to the file of noncritical code (File 3 in Figure 6)

If the critical count exceeds the chosen critical limit

Begin

Print a warning message to the screen or analysis file.

Allow the investigator/programmer to change the code or respecify the critical limit.

Start the process over from the beginning.

End

If the noncritical count exceeds the noncritical limit

Begin

Print a warning message to the screen or analysis file.

Allow the investigator/programmer to

(1) Change the noncritical limit.

(2) Request that the state be broken in two at the point of error.

(3) Ignore the error and allow this state to exEnd ceed the noncritical limit.

Loop to the beginning of these instructions until all of the user code has been read and processed.

End Algorithm

Once the preprocessor has finished running, three files will have been generated. One is the analysis of execu- tion times for the user-written code. The second is a file of functions that have the same names as do the states in the user program. This file is the file of noncritical code; it looks much like a regular program, except that there are address labels instead of function names. The third file is a file of critical processing routines that will consist of all of the code that the investigator specified as timecritical. This second file consists of interrupt service routines that must be linked with the first file; then, the whole program may be compiled and run.

\section{Conclusion}

When computers used for on-line control of experiments had small memories, it was important to write programs that used the limited space efficiently; when the central processing units were slow, it was important to write programs that used time efficiently. Now that the computers that are used to control experiments have memories measured in megabytes and processor speeds measured in tens of megahertz, it has become important to write programs that are reliable and analyzable. Our system is such a program. It enables us to write a wide variety of programs, including real-time experiment control programs, in such a way that we are assured that critical events are not missed.

\section{REFERENCES}

Aho, A., Ullman, J. (1977). Principles of compiler design. Reading, MA: Addison-Wesley.

Dyckman, J. L., \& Church, R. M. (1972). The TIICIE System for Interactive Control of Independent Experiments. Computers in the Psychology Laboratory, 2, 27-34.

Foster, C. C. (1981). Real-time programming: Neglected topics. Reading, MA: Addison-Wesley.

TANEnbaum, A. S. (1987). Operating systems: Design and implementation. Englewood Cliffs, NJ: Prentice-Hall.

Young, S. J. (1982). Real-time languages, design, and development. East Horwood, NY: Halsted Press.

\section{NOTES}

1. For example, from Ryle Design, P.O. Box 22, Mt. Pleasant, MI 48804, CompuServe 73047,1765.

2. Our state-transition system was based on an earlier system developed over several years by Russell Church, Howard Dyckman, Robert Moore, and Nick Staddon. Our first system, written in LINC assembly language and executed by a DEC PDP- 12 computer, used clock-based polling with a single user-defined time base for both critical and noncritical processing, which was usually set at $100 \mathrm{msec}$. The user-written code was similar to that currently being used, but the preprocessor did not analyze the time requirements. The worst case actually encountered was available during the running of the program. (See Dyckman \& Church, 1972.)

3. Source code ( 65 pages) and documentation of our current system and associated utilities are available.

4. Both the operating systems book by Tanenbaum (1987) and the compiler design book by Aho and Ullman (1977) are excellent. Both are fairly easy to read, even if one has minimal experience programming in $\mathrm{C}$. They provide much more information about analysis of realtime control programs and language analysis than the length of this paper allows. They also explore other solutions to some of the problems of reliability that are not described in this paper. Much of our code was inspired by these two books. Two other excellent books on real-time programming are: Foster (1981) and Young (1982). 